\title{
The Existence of Non-Topological Solitons in the Self-Dual Chern-Simons Theory
}

\author{
Joel Spruck ${ }^{1, \star}$ and Yisong Yang ${ }^{2}$ \\ 1 Department of Mathematics, University of Massachusetts, Amherst, MA 01003, USA and I.H.E.S, \\ Bures-Sur-Yvette, France \\ 2 Department of Mathematics and Statistics, University of New Mexico, Albuquerque, NM 87131, \\ USA
}

Received December 6, 1991; in revised form March 10, 1992

\begin{abstract}
In the recently discovered $(2+1)$-dimensional relativistic Chern-Simons model, self-duality can be achieved when the Higgs potential density assumes a special form for which both the asymmetric and symmetric vacua are ground state solutions. This important feature may imply the coexistence of static topological and non-topological vortex-like solutions in $\mathbb{R}^{2}$ but the latter have been rather elusive to a rigorous construction. Our main purpose in this paper is to prove the existence of non-topological radially symmetric $N$-vortex solutions in the self-dual Chern-Simons model. By a shooting method, we obtain a continuous family of gauge-distinct $N$ vortex solutions. Moreover, we are also able to classify all possible bare (or 0-vortex) solutions.
\end{abstract}

\section{Introduction}

Although it has long been speculated that the addition of a Chern-Simons term to the usual Yang-Mills-Higgs action density in $(2+1)$ dimensions would lead to static finite energy vortex-like solutions that carry both electric and magnetic charges, it is the work of Hong, Kim, and Pac [10] and Jackiw and Weinberg [15] (see also Lee [17] for the nonabelian case) which enables one to make a rigorous study of such solutions. The crucial feature in their approach is that, when the Yang-Mills (or Maxwell) term is dropped from the Lagrangian and the Higgs potential takes on a special form, the static equations of motion can be reduced to a new Bogomol'nyi type system of first order equations. Due to the form of the Higgs potential, both topological and non-topological solutions may be present [11-14]. In fact, the system shares some common properties with the Bogomol'nyi equations arising from the abelian Higgs model [5] and hence, partial understanding may be achieved using some techniques developed earlier. In particular, Wang [25] has proved the existence of topological multivortex solutions by the variational method in Jaffe and Taubes [16]. Moreover,

* Research supported in part by NSF grant DMS-88-02858 and DOE grant DE-FG02-86ER250125 
we have shown [22] that topological multivortex solutions can actually be constructed by a globally convergent iterative method, which may be implemented efficiently in practical numerical computations, and that our solutions are most superconducting. However, non-topological solutions are much more difficult to obtain than topological ones. For example, the structure of the equations and the lack of a suitable function space setting makes it forbidding to formulate a variational problem. Even in the radical case, the method of Tyupkin, Fateev, and Shvarts [24], used so successfully in the study of existence of solutions in gauge field theories, does not seem to be applicable here.

In this paper, we shall construct non-topological radially symmetric $N$-vortex solutions in the self-dual Chern-Simons model proposed by Jackiw et al. We will view the reduced ordinary differential equation as a dynamical system and use a shooting argument to prove the existence of a continuous family of gauge-distinct solutions. We are also able to find all possible bare (or 0-vortex) solutions. Our method here may be suggestive to some other problems in this and other related areas.

The contents of the paper are as follows. In Sect. 2 we introduce the problems that we are going to study and then state our main results. In Sects. 3-5 we present our proofs. Finally we complete the paper in Sect. 6 with some concluding remarks.

\section{Statements of Main Results}

We shall follow the notation in Jackiw and Weinberg [15]. The $(2+1)$-dimensional Minkowski space metric tensor $g_{\mu \nu}$ is diag $(1,-1,-1)$. Assuming normalized units and the critical coupling, the Lagrangian action density of the Chern-Simons model is given by the expression

$$
\mathscr{L}=\left(D_{\mu} \phi\right)\left(D^{\mu} \phi\right)^{*}+\frac{1}{4} \kappa \varepsilon^{\alpha \beta \gamma} A_{\alpha} F_{\beta \gamma}-\frac{1}{\kappa^{2}}|\phi|^{2}\left(1-|\phi|^{2}\right)^{2},
$$

where $\phi$ is a complex scalar field, $A_{\mu}(\mu=0,1,2)$ a vector field, $D_{\mu} \phi=\partial_{\mu} \phi-i A_{\mu} \phi$, $\kappa>0$ a constant, $\varepsilon^{\alpha \beta \gamma}$ the Levi-Civita totally skew-symmetric tensor with $\varepsilon^{012}=1$, and $F_{\mu \nu}=\partial_{\mu} A_{\nu}-\partial_{\nu} A_{\mu}$.

The Euler-Lagrange equations of (2.1) read

$$
\left\{\begin{array}{l}
\frac{1}{2} \kappa \varepsilon^{\alpha \beta \gamma} F_{\beta \gamma}=i\left(\phi\left[D^{\alpha} \phi\right]^{*}-\phi^{*}\left[D^{\alpha} \phi\right]\right), \\
D_{\mu} D^{\mu} \phi=-\frac{1}{\kappa^{2}}\left(2|\phi|^{2}\left[|\phi|^{2}-1\right]+\left[|\phi|^{2}-1\right]^{2}\right) \phi .
\end{array}\right.
$$

We will only be interested in the static solutions of (2.2). Hence the $\alpha=0$ component of the first equation in (2.2) implies the Chern-Simons Gauss law

$$
\kappa F_{12}=-2 A_{0}|\phi|^{2} \text {. }
$$

Inserting (2.3) into the $(0,0)$-component of the energy-momentum tensor

$$
T_{\mu \nu}=2 \operatorname{Re}\left\{\left(D_{\mu} \phi\right)^{*}\left(D_{\nu} \phi\right)\right\}-g_{\mu \nu}\left[\left(D_{\gamma} \phi\right)\left(D^{\gamma} \phi\right)^{*}-\frac{1}{\kappa^{2}}|\phi|^{2}\left(1-|\phi|^{2}\right)^{2}\right]
$$

it is seen that the energy density is

$$
\mathscr{E}=\frac{\kappa^{2}}{4} \cdot \frac{F_{12}^{2}}{|\phi|^{2}}+\left|D_{j} \phi\right|^{2}+\frac{1}{\kappa^{2}}|\phi|^{2}\left(1-|\phi|^{2}\right)^{2},
$$

where $j=1,2$. Hong, Kim, and Pac [10] and Jackiw and Weinberg [15] first showed 
via (2.4) that (2.2) allows a reduction to the first order Bogomol'nyi system

$$
\left\{\begin{array}{l}
D_{1} \phi+i D_{2} \phi=0 \\
F_{12}+\frac{2}{\kappa^{2}}|\phi|^{2}\left(|\phi|^{2}-1\right)=0
\end{array}\right.
$$

coupled with Eq. (2.3).

The form of the energy density (2.4) implies that a finite energy solution of (2.5) verifies either

$$
|\phi(x)| \rightarrow 1 \quad \text { as } \quad|x| \rightarrow \infty
$$

or

$$
|\phi(x)| \rightarrow 0 \text { as }|x| \rightarrow \infty .
$$

The former is called topological which gives rise to quantized magnetic and electric charges, etc., while the latter is called non-topological [11-14] for which the charges may be fractal. (However, at this moment, such a statement remains more or less a conjecture. Unlike topological solutions, the charges of non-topological solutions depend on their accurate decay rates at infinity which are rather difficult to obtain. One of the most interesting features of these solutions is that they carry more than twice as much as the charges of a topological solution realizing a given vortex number. See Remark i) in Sect. 6 for details.) Topological multivortex solutions are already well understood. The purpose of this paper is to establish some existence results for non-topological solutions.

Concerning $N$-vortex solutions, we have

Theorem 2.1. For any $\tilde{x} \in \mathbb{R}^{2}$ and a given integer $N \geqq 1$ and any $\alpha \geq \ln 2$, the Bogomol' nyi equations (2.5) have a finite energy solution $\left(\phi^{(\alpha)}, A^{(\alpha)}\right)$ so that the only zero of $\phi^{(\alpha)}$ is $x=\tilde{x}$ and the multiplicity of the zero is $N$. Moreover, $\left(\phi^{(\alpha)}, A^{(\alpha)}\right)$ is radially symmetric about the point $x=\tilde{x}$ and

$$
\begin{gathered}
\max _{x \in \mathbb{R}^{2}}\left|\phi^{(\alpha)}(x)\right|^{2}=e^{-\alpha}, \\
\left|\phi^{(\alpha)}(x)\right|^{2}=O\left(r^{-\sigma}\right) \text { for large } r=|x|,
\end{gathered}
$$

where $\sigma>2 N+4$ is a constant which may depend on $\alpha$ and $\tilde{x}$.

Since (2.7) is gauge-invariant, we see that different values of $\alpha$ give rise to gaugedistinct solutions. In particular, there is non-uniqueness.

Let $(\phi, A)$ be a finite energy solution pair of (2.5). If $\phi$ is nonvanishing in $\mathbb{R}^{2}$, the solution is called a bare (or 0-vortex) solution following Jackiw, Lee, Pi, and Weinberg $[12,14]$. For bare solutions, we have the following description.

Theorem 2.2. If $(\phi, A)$ is a finite energy solution of (2.5)-(2.6) so that $\phi \neq 0$ in $\mathbb{R}^{2}$, then $(\phi, A)$ is necessarily radially symmetric about some point $\tilde{x} \in \mathbb{R}^{2}$ and

$$
|\phi(\tilde{x})|^{2}=\max _{x \in \mathbb{R}^{2}}|\phi(x)|^{2}, \quad|\phi(\tilde{x})|^{2}<1
$$

and $|\phi(x)|^{2}$ is a strictly decreasing function of $r=|x-\tilde{x}|$. Moreover, for any $\tilde{x} \in \mathbb{R}^{2}$ and $\alpha>0$, the system (2.5)-(2.6) has a unique solution $\left(\phi^{(\alpha)}, A^{(\alpha)}\right)$ which is radially symmetric about $\tilde{x}$ and

$$
\begin{gathered}
\max _{x \in \mathbb{R}^{2}}\left|\phi^{(\alpha)}(x)\right|^{2}=\left|\phi^{(\alpha)}(\tilde{x})\right|^{2}=e^{-\alpha}, \\
\left|\phi^{(\alpha)}(x)\right|^{2}=O\left(r^{-\sigma}\right) \quad \text { for large } r=|x|,
\end{gathered}
$$

where $\sigma>4$ is a constant which may depend on $\alpha$ and $\tilde{x}$. 
It is obvious that any nonvanishing topological solutions of (2.5) must be trivial: $|\phi|=1, A=0$ (see the discussion in the first paragraph of Sect. 4). Therefore we have found that all possible bare solutions of the self-dual Chern-Simons equations (2.5). As for the $N$-vortex solutions (see Theorem 2.1), this is also a three-parameter continuous family of gauge-distinct non-topological solutions, labelled by $\alpha>0$ and $\tilde{x} \in \mathbb{R}^{2}$.

The above theorems will be proved in the subsequent sections.

\section{Existence of Vortices via Shooting}

Since (2.5) is invariant under space translations, we may assume that $\tilde{x}$ is the origin. Therefore we shall look for a solution $(\phi, A)$ of $(2.5)-(2.6)$ so that $\phi$ has the local property

$$
|\phi(x)|=|x|^{N} \eta(x) \quad \text { near } x=0,
$$

where $N \geq 1$ is a given integer and $\eta(x)$ is a nonvanishing function. In view of (3.1), it is standard that the substitution $u=\ln |\phi|^{2}$ reduces (2.5) to the elliptic equation

$$
\Delta u=\frac{4}{\kappa^{2}} e^{u}\left(e^{u}-1\right)+4 \pi N \delta(x), \quad x \in \mathbb{R}^{2},
$$

where $\delta(x)$ is the Dirac distribution. Let $v=u-N \ln |x|^{2}$. Then (3.2) becomes

$$
\Delta v=\frac{4}{\kappa^{2}}|x|^{2 N} e^{v}\left(|x|^{2 N} e^{v}-1\right), \quad x \in \mathbb{R}^{2},
$$

We will restrict our attention to radially symmetric solutions of (3.3): $v=v(r)$, $r=|x|>0$. Hence (3.3) takes on the form

$$
v_{r r}+\frac{1}{r} v_{r}=\frac{4}{\kappa^{2}} r^{2 N} e^{v}\left(r^{2 N} e^{v}-1\right), \quad r>0 .
$$

Eventually we want to extend a solution of (3.4) defined in $\mathbb{R}^{2}-\{0\}$ to recover a smooth solution of (3.3) in full $\mathbb{R}^{2}$ (so that $u$ is asymptotic to $2 N \ln |x|$ as $x$ tends to zero). For this purpose, we need the following special form of the well-known removable singularity theorem [21]:

Lemma 3.1. Let $\Omega$ be a domain in $\mathbb{R}^{2}$ containing the origin $x=0$ and $f$ a harmonic function defined in the punctured domain $\Omega-\{0\}$. Then $f$ can be extended to a harmonic function in $\Omega$ if and only if

$$
\lim _{|x| \rightarrow 0} \frac{f(x)}{\ln |x|}=0 .
$$

Using Lemma 3.1 in our problem, we have

Lemma 3.2. The solution $v$ of (3.4) can be extended to a smooth solution of (3.3) in $\mathbb{R}^{2}$ if and only if

$$
\lim _{r \rightarrow 0} \frac{v(r)}{\ln r}=0 .
$$

Proof. Let $v(r)$ be a solution of (3.4) verifying (3.6). Then for any $a>0$, we have

$$
\lim _{r \rightarrow 0} r^{a} e^{v(r)}=\lim _{r \rightarrow 0} e^{\ln r\left(a+\frac{v(r)}{\ln r}\right)}=0 .
$$


Thus it is easily seen that the right-hand side of (3.4) can be viewed as a Hölder continuous function over the full $\mathbb{R}^{2}$.

Let $w$ be a solution of

$$
\Delta w=\frac{4}{\kappa^{2}} r^{2 N} e^{v}\left(r^{2 N} e^{v}-1\right)
$$

in a small neighborhood of the origin, say $\Omega$. Then $w$ is $C^{2}$-Hölder continuous and $f=v-w$ is harmonic in $\Omega-\{0\}$. However, (3.6) says that $f$ fulfills the condition (3.5). Therefore, using Lemma 3.1, we see that $v$ is $C^{2}$-Hölder continuous in $\Omega$. A bootstrap argument then shows that $v$ is $C^{\infty}$ in $\mathbb{R}^{2}$.

Thus from now on we shall look for solutions of (3.4) under the condition (3.6). It will be most convenient to study the equation in the original variable $u(r)=$ $N \ln r^{2}+v(r)$ [see (3.2)]. Hence (3.4) is changed into the simpler form

$$
u_{r r}+\frac{1}{r} u_{r}=\frac{4}{\kappa^{2}} e^{u}\left(e^{u}-1\right) \quad r>0 .
$$

The boundary condition (3.6) now becomes

$$
\lim _{r \rightarrow 0} \frac{u(r)}{\ln r}=2 N
$$

Recall that we need to find non-topological solutions of the Bogomil'nyi system (2.5)-(2.6). Therefore the relation $|\phi|^{2}=e^{u}$ implies that $u$ is subject to the following boundary condition at $r=\infty$ :

$$
\lim _{r \rightarrow \infty} u(r)=-\infty
$$

Our goal now is to find solutions of (3.7) under the boundary constraints (3.8)(3.9). We shall show that for suitable $r_{0}>0$, we can obtain global solutions of (3.7) coupled with some adequate initial data at $r=r_{0}$ to fulfill (3.8)-(3.9). In other words, we are going to solve (3.7)-(3.9) by a two-side shooting technique.

To motivate our shooting data, we first make a simple observation.

Lemma 3.3. If $u(r)$ is a solution of (3.7) satisfying

$$
\lim _{r \rightarrow 0} u(r)=-\infty, \quad \lim _{r \rightarrow \infty} u(r)=-\infty,
$$

then $u(r)<0$ for all $r>0$.

Proof. The conclusion can be seen directly from a maximum principle argument.

From Lemma 3.3 we see that a desired solution of (3.7)-(3.9) must have a global maximum $u_{0}=-\alpha<0$ at some $r=r_{0}>0$. Therefore we should look for solutions of (3.7) under the initial condition

$$
u\left(r_{0}\right)=-\alpha, \quad u_{r}\left(r_{0}\right)=0 .
$$

We expect that, when $r_{0}>0, \alpha>0$ are suitably chosen, the unique solution of (3.7) under the condition (3.10) will verify both (3.8)-(3.9). Our study in this section shows that such a goal can be achieved.

To simplify the discussion, we introduce a change of independent variable

$$
t=\ln r, \quad t_{0}=\ln r_{0} .
$$


Then (3.7) and (3.10) become

$$
\left\{\begin{array}{l}
u^{\prime \prime}=\frac{4}{\kappa^{2}} e^{2 t} e^{u}\left(e^{u}-1\right), \quad-\infty<t<\infty \\
u\left(t_{0}\right)=-\alpha \\
u^{\prime}\left(t_{0}\right)=0
\end{array}\right.
$$

where, and in the sequel, $u^{\prime}=d u / d t$ and $u(t)$ denotes the dependence of the solution $u$ of (3.7) on the new variable $t$ (or vice versa, for simplicity).

Lemma 3.4. For any $t_{0} \in \mathbb{R}$ and $\alpha>0$, (3.12) has a unique global solution $u(t)$. This solution satisfies $u(t)<0$ and

$$
\lim _{t \rightarrow-\infty} u(t)=-\infty, \quad \lim _{t \rightarrow \infty} u(t)=-\infty
$$

Proof. Let $u(t)$ be a local solution of (3.12). Then in the interval of existence,

$$
u^{\prime}(t)=\frac{4}{\kappa^{2}} \int_{t_{0}}^{t} e^{2 s} e^{u(s)}\left(e^{u(s)}-1\right) d s .
$$

We can show that, for all $t$, where $u(t)$ exists, there holds $u(t)<0$. In fact, if there is a $\bar{t}>t_{0}$ so that $u(\bar{t}) \geq 0$, we may assume $\bar{t}$ is such that

$$
\bar{t}=\inf \left\{t \geq t_{0} \mid u(t) \text { exists and } u(t) \geq 0\right\} .
$$

Then $\bar{t}>t_{0}$ and $u(\bar{t})=0$. Obviously $u(t)<0$ for all $t_{0} \leq t<\bar{t}$. However, from (3.14), we see that $u^{\prime}(t)<0$ for $t_{0}<t \leq \bar{t}$. So $u(\bar{t})<0$. This reaches a contradiction.

Similarly, if there is a $\bar{t}<t_{0}$ so that $u(\bar{t})=0$ and $u(t)<0$ for $\bar{t}<t \leq t_{0}$, then $u^{\prime}(t)>0$ for $\bar{t} \leq t<t_{0}$. So $u(\bar{t})<0$. This is again a contradiction.

From the property $u(t)<0$ and (3.14), it is seen that $u^{\prime}(t)$ cannot blow up in finite time. As a consequence the solution of (3.12) exists globally in $t \in(-\infty, \infty)$.

The behavior $u(t) \rightarrow-\infty$ as $t \rightarrow-\infty$ is easy to verify because $u(t)<0$ and (3.14) imply that

$$
\lim _{t \rightarrow-\infty} u^{\prime}(t)=\frac{4}{\kappa^{2}} \int_{t_{0}}^{-\infty} e^{2 s} e^{u(s)}\left(e^{u(s)}-1\right) d s=C>0 .
$$

Finally we show that $u(t) \rightarrow-\infty$ as $t \rightarrow \infty$. By virtue of $u(t)<0$ and (3.14), we have $u^{\prime}(t)<0$ for $t>t_{0}$. Therefore, either $u(t) \rightarrow-\infty$ or $u(t) \rightarrow$ a finite number $a<-\alpha<0$ as $t \rightarrow \infty$. However, the latter situation cannot happen. To see this, we assume otherwise. Thus by $a<u(t) \leq-\alpha$ and (3.14) we find the estimate

$$
\begin{aligned}
u^{\prime}(t) & \geq \frac{4}{\kappa^{2}} \int_{t_{0}}^{t} e^{2 s}\left[\min _{a \leq u \leq-\alpha}\left\{e^{u}\left(e^{u}-1\right)\right\}\right] d s \\
& =-C \int_{t_{0}}^{t} e^{2 s} d s=-\frac{C}{2}\left(e^{2 t}-e^{2 t_{0}}\right), \quad t>t_{0},
\end{aligned}
$$

where $C>0$ is a constant. A simple consequence of (3.15) is that $u(t) \rightarrow-\infty$ as $t \rightarrow \infty$. This contradicts our assumption. 
In terms of the new variable $t$ [see (3.11)], the boundary condition (3.8) reads

$$
\lim _{t \rightarrow-\infty} \frac{u(t)}{t}=2 N
$$

Lemma 3.5. For any given $\alpha \geq \ln 2$, there is a $t_{0}=t_{0}(\alpha)$ such that the unique solution of (3.12) verifies the condition (3.16).

Proof. For $t_{0} \in(-\infty, \infty)$ and $\alpha>0$, let $u=u\left(t ; t_{0}, \alpha\right)$ be the unique global solution of (3.12). Then $u<0$ and $u \rightarrow-\infty$ as $t \rightarrow-\infty$ by Lemma 3.4. Therefore, using the L'Hôpital rule, (3.16) reads

$$
\eta\left(t_{0}, \alpha\right) \equiv \lim _{t \rightarrow-\infty} u^{\prime}\left(t ; t_{0}, \alpha\right)=2 N
$$

where, in view of $(3.14)$, the function $\eta\left(t_{0}, \alpha\right)$ has the representation

$$
\eta\left(t_{0}, \alpha\right)=-\frac{4}{\kappa^{2}} \int_{-\infty}^{t_{0}} e^{2 s} e^{u\left(s ; t_{0}, \alpha\right)}\left(e^{u\left(s ; t_{0}, \alpha\right)}-1\right) d s .
$$

Since $u<0$ and $u$ depends continuously on $t_{0}, \alpha$, (3.18) says that $\eta$ is a continuous function of $t_{0}, \alpha$. In the following, we shall show that there are $t_{0}, \alpha$ to make $\eta$ fulfill the condition (3.17).

Step 1. From (3.12) we get $u^{\prime \prime}>-\left(4 / \kappa^{2}\right) e^{2 t} e^{u}$. Set $w=2 t+u$. Then

$$
w^{\prime \prime}>-\frac{4}{\kappa^{2}} e^{w}
$$

However, since $u^{\prime} \geq 0$ for $t \leq t_{0}$, we have $w^{\prime}>0$ when $t \leq t_{0}$. Multiplying (3.19) by $w^{\prime}$ and integrating on $\left(t, t_{0}\right)$, we find

$$
4-\left(w^{\prime}(t)\right)^{2}>\frac{8}{\kappa^{2}}\left(e^{w(t)}-e^{2 t_{0}-\alpha}\right), \quad t<t_{0},
$$

namely,

$$
0<u^{\prime}\left(t ; t_{0}, \alpha\right)<2 \sqrt{1+\frac{2}{\kappa^{2}} e^{2 t_{0}-\alpha}}-2 \equiv K, \quad t<t_{0} .
$$

From (3.20), we obtain another useful inequality

$$
-\alpha>u\left(t ; t_{0}, \alpha\right)>-\alpha-K\left(t_{0}-t\right), \quad t<t_{0} .
$$

Step 2. It is straightforward to examine that $e^{u}\left(e^{u}-1\right)$ is a decreasing function in $u \in(-\infty,-\ln 2]$. Therefore the condition $\alpha \geq \ln 2$, (3.21), and (3.12) imply

$$
u^{\prime \prime}<\frac{4}{\kappa^{2}} e^{2 t} e^{-\alpha-K\left(t_{0}-t\right)}\left(e^{-\alpha-K\left(t_{0}-t\right)}-1\right), \quad t<t_{0} .
$$

Integrating (3.22) over $\left(-\infty, t_{0}\right)$ gives

$$
\begin{aligned}
-\eta\left(t_{0}, \alpha\right) & =-\lim _{t \rightarrow-\infty} u^{\prime}\left(t ; t_{0}, \alpha\right) \\
& \leq \frac{4}{\kappa^{2}} \int_{-\infty}^{t_{0}} e^{2 s} e^{-\alpha-K\left(t_{0}-s\right)}\left(e^{-\alpha-K\left(t_{0}-s\right)}-1\right) d s
\end{aligned}
$$


Or

$$
\begin{aligned}
\eta\left(t_{0}, \alpha\right) & \geq \frac{4}{\kappa^{2}} e^{2 t_{0}-\alpha}\left(\frac{1}{K+2}-\frac{e^{-\alpha}}{2 K+2}\right) \\
& \geq \frac{4}{\kappa^{2}} e^{2 t_{0}-\alpha}\left(\frac{1}{K+2}-\frac{1}{2(2 K+2)}\right) \\
& \geq \frac{2 e^{2 t_{0}-\alpha}}{\kappa^{2}(K+2)}=e^{2 t_{0}-\alpha} / \kappa \sqrt{\kappa^{2}+2 e^{2 t_{0}-\alpha}} .
\end{aligned}
$$

Step 3. In view of (3.20), we have

$$
0<\eta\left(t_{0}, \alpha\right) \leq 2 \sqrt{1+\frac{2}{\kappa^{2}} e^{2 t_{0}-\alpha}}-2 .
$$

Therefore, for any $\alpha \geq \ln 2>0$, we can find a suitable $t_{0}=t_{0}^{\prime}$ so that $\eta\left(t_{0}^{\prime}, \alpha\right)<2 N$. On the other hand, (3.23) says that for fixed $\alpha \geq \ln 2$, there is some $t_{0}=t_{0}^{\prime \prime}$ to make $\eta\left(t_{0}^{\prime \prime}, \alpha\right)>2 N$. Consequently, there is a point $t_{0}=t_{0}(\alpha)$ between $t_{0}^{\prime}$ and $t_{0}^{\prime \prime}$ so that $\eta\left(t_{0}, \alpha\right)=2 N$.

We next study the asymptotic behavior of the solution $u(t)$ of (3.12) (as $t \rightarrow \infty$ ) produced in Lemma 3.5. We have

Lemma 3.6. There is a constant $\beta>2 N+4$ so that

$$
\lim _{t \rightarrow \infty} u^{\prime}(t)=-\beta \text {. }
$$

Proof. Since $u^{\prime \prime}<0$ [see (3.12) and Lemma 3.4], we see that either $u^{\prime}(t) \rightarrow-\infty$ or a finite number as $t \rightarrow \infty$. First suppose that $u^{\prime}(t) \rightarrow-\infty$ as $t \rightarrow \infty$. Then there is a $\bar{t}$ so that $u^{\prime}(t)<-3$ (say) for $t>\bar{t}$. Hence $u(t)<-3 t+C\left(t>t_{0}\right)$ for some constant $C$ and

$$
\begin{aligned}
\lim _{t \rightarrow \infty} u^{\prime}(t)= & \frac{4}{\kappa^{2}} \int_{t_{0}}^{\infty} e^{2 s} e^{u(s)}\left(e^{u(s)}-1\right) d s \\
& >-\frac{4}{\kappa^{2}} \int_{t_{0}}^{\infty} e^{2 s} e^{-3 s+C} d s=-\frac{4}{\kappa^{2}} e^{C-t_{0}}>-\infty
\end{aligned}
$$

which is a contradiction.

Thus, in the sequel, we assume there is a $\beta>0$ to make (3.24) hold. It remains to show that $\beta>2 N+4$.

First of all, since $u^{\prime}(t)$ is decreasing for $t \geq t_{0}$, therefore $u^{\prime}(t)>-\beta, t \geq t_{0}$ and $u(t)>-\beta t+C, t \geq t_{0}$, where $C$ is a constant. It is obvious that (3.14) and (3.24) imply the convergence of the integral

$$
\int_{t_{0}}^{\infty} e^{2 s} e^{u(s)} d s
$$

As a consequence, we must have $\beta>2$. Such a property in turn implies that

$$
\lim _{t \rightarrow \infty} e^{2 t} e^{u(t)}=\lim _{t \rightarrow \infty} e^{t\left(2+\frac{u(t)}{t}\right)}=0
$$


Next, multiplying the first equation in (3.12) by $u^{\prime}$, integrating over $(-\infty, \infty)$, and using (3.25), we obtain

$$
\beta^{2}-4 N^{2}=-\frac{16}{\kappa^{2}} \int_{-\infty}^{\infty} e^{2 s} e^{u(s)}\left(e^{u(s)}-1\right) d s+\frac{8}{\kappa^{2}} \int_{-\infty}^{\infty} e^{2 s} e^{2 u(s)} d s
$$

Namely

$$
\beta^{2}-4 \beta-2 N(2 N+4)=\frac{8}{\kappa^{2}} \int_{-\infty}^{\infty} e^{2 s} e^{2 u(s)} d s>0 .
$$

Therefore $\beta>2 N+4$ as desired.

Let $u$ be the solution of (3.12) satisfying the properties stated in Lemmas 3.5-3.6. Thus, in terms of the original variable $r=e^{t}$, the function $u$ is a solution of (3.7)(3.9). Thus, from the earlier discussion, it is seen that $u$ is in fact a radially symmetric classical solution of the $N$-vortex equation (3.2). In $\mathbb{R}^{2}=\mathbb{C}$, set

$$
z=x_{1}+i x_{2}, \quad \partial=\left(\partial_{1}-i \partial_{2}\right) / 2, \quad \partial^{*}=\left(\partial_{1}+i \partial_{2}\right) / 2 \text {. }
$$

Define

$$
\begin{gathered}
\theta(z)=2 N \arg (z), \quad \phi(z)=\exp \left(\frac{1}{2}[u(z)+i \theta(z)]\right), \\
A_{1}(z)=-\operatorname{Re}\left\{2 i \partial^{*} \ln \phi(z)\right\}, \quad A_{2}(z)=-\operatorname{Im}\left\{2 i \partial^{*} \ln \phi(z)\right\} .
\end{gathered}
$$

Then $(\phi, A)$ is a solution of the Bogomol'nyi equations (2.5) so that $\phi(0)=0$, the multiplicity of this zero is $N$, and $\phi$ is nonvanishing elsewhere.

Since $u$ is globally strictly concave in $r>0$ and $\max u(r)=u\left(r_{0}\right)$, we have

$$
\max _{x \in \mathbb{R}^{2}}|\phi(x)|^{2}=e^{u\left(r_{0}\right)}=e^{-\alpha} \text {. }
$$

This yields (2.7).

Next, to see the asymptotic behavior of $\phi$, we proceed as follows.

Let $\sigma$ be such that $2 N+4<\sigma<\beta$ (see Lemma 3.6). Then

$$
\frac{|\phi(x)|^{2}}{r^{-\sigma}}=r^{\sigma} e^{u}=e^{t\left(\frac{u(t)}{t}+\sigma\right)},
$$

where $r=|x|$ and $t=\ln r$. However, since

$$
\lim _{t \rightarrow \infty} \frac{u(t)}{t}=\lim _{t \rightarrow \infty} u^{\prime}(t)=-\beta
$$

and $\beta>\sigma$, therefore as $t \rightarrow \infty$ the right-hand side of (3.27) goes to zero. This proves that $\phi$ satisfies (2.8).

Finally we show that the solution pair $(\phi, A)$ is of finite energy. In fact a simple calculation gives

$$
D_{1} \phi=\left(\partial_{1} u-i \partial_{2} u\right) \phi, \quad D_{2} \phi=\left(i \partial_{1} u+\partial_{2} u\right) \dot{\phi} .
$$

Thus the second term in (2.4) has the representation $\left|D_{j} \phi\right|^{2}=2 u_{r}^{2} e^{u}, r=|x|$. However, from the relation $r u_{r}=u^{\prime}(t)$ and Lemma 3.6 we have $u_{r}=O\left(r^{-1}\right)$ for large $r>0$. As a consequence,

$$
\left|D_{j} \phi\right|^{2}=O\left(r^{-(\sigma+2)}\right) \quad \text { for large } r=|x|>0 .
$$


Hence $\int\left|D_{j} \phi\right|^{2} d x<\infty$. Moreover, from the decay estimate (2.8) and (2.5), we see immediately that the other two terms in (2.4) also have finite integrals over $\mathbb{R}^{2}$. This proves the finiteness of the energy.

The proof of Theorem 2.1 is complete.

\section{Proof of Symmetry for Bare Solutions}

Let $(\phi, A)$ be a solution pair of (2.5). If $\phi \neq 0$ in $\mathbb{R}^{2}$, then the substitution $u=\ln |\phi|^{2}$ transforms (2.5) into the elliptic equation

$$
\Delta u=\frac{4}{\kappa^{2}} e^{u}\left(e^{u}-1\right), \quad x \in \mathbb{R}^{2} .
$$

In fact (4.1) corresponds to the $N=0$ sector of (3.2). An easy application of the maximum principle shows that if $u$ gives rise to a topological solution $(\phi, A)$ so that $u=0$ at infinity, then $u \equiv 0$ in $\mathbb{R}^{2}$ because the structure of (4.1) says that a solution of the equation cannot have a positive maximum or a negative minimum in $\mathbb{R}^{2}$. Thus we will only be interested in non-topological solutions of (4.1) which satisfy

$$
\lim _{|x| \rightarrow \infty} u(x)=-\infty
$$

We shall show that any solution of (4.1)-(4.2) must be radially symmetric about some point in $\mathbb{R}^{2}$. In fact, our result can be established under the slightly weaker condition

$$
\int_{\mathbb{R}^{2}} e^{u}\left(1-e^{u}\right) d x<\infty .
$$

In other words, we will prove that any solution of (4.1) satisfying (4.3) is necessarily radially symmetric.

The study of the symmetry properties of solutions of the equation $\Delta u=f(u)$ in $\mathbb{R}^{n}(n \geq 3)$ has a rich history beginning with the work of Gidas, Ni, and Nirenberg [8]. Many of the results of [8] were simplified and improved by $\mathrm{Li}$ [18] (see also the recent paper [4]). One interesting special case of [8] says that when $f(u)=-u^{p}$ and $p=(n+2) /(n-2)$, a positive solution of the equation decaying at infinity sufficiently fast must be radially symmetric about some point in $\mathbb{R}^{n}$. This result was improved by Caffarelli, Gidas, and Spruck [6] who showed the same symmetry property without any decay assumption. Recently, Chen and Li [7] studied the symmetry problem for the equation in $\mathbb{R}^{2}$ with $f(u)=-e^{u}$ (the completely integrable Liouville equation [19]). This is the natural analogue for $n=2$ of the critical nonlinearity $f(u)=-u^{p}$ with $p=(n+2) /(n-2)$ when $n>2$ studied in [6]. Chen and Li showed that a solution satisfying $\int_{\mathbb{R}^{2}} e^{u} d x<\infty$ must be radially symmetric about some point in $\mathbb{R}^{2}$. In this section, we present an adaptation of their work to Eq. (4.1) under the assumption (4.3). Note that no nontrivial explicit analytic solution of (4.1) is known.

We first observe in the following lemma that if $u \neq \equiv 0$ is a solution of (4.1), then necessarily $u<0$ in $\mathbb{R}^{2}$. In particular the condition (4.3) makes good sense.

Lemma 4.1. Let $u \neq \equiv$ be a global $C^{2}$-solution of (4.1). Then $u<0$ in $\mathbb{R}^{2}$.

Proof. We first show that a radial solution $v=v(r)$ of (4.1) with $v(0)=\alpha>0$ and $v^{\prime}(0)=0$ goes to $\infty$ at some finite radius $R=R(\alpha)$. 
Consider

$$
\left\{\begin{array}{l}
v_{r r}+\frac{1}{r} v_{r}=\frac{4}{\kappa^{2}} e^{v}\left(e^{v}-1\right), \quad r>0 \\
v(0)=\alpha \\
v_{r}(0)=0
\end{array}\right.
$$

It is well-known that (4.4) has a unique local solution for any given $\alpha \in \mathbb{R}$ and this solution gives rise to a solution of (4.1) in a neighborhood of the origin. See for example Berestycki, Lions, and Peletier [3]. As in Sect. 3, the substitution (3.11) transforms (4.4) into

$$
\left\{\begin{array}{l}
v^{\prime \prime}=\frac{4}{\kappa^{2}} e^{2 t} e^{v}\left(e^{v}-1\right), \quad-\infty<t<\infty \\
v(t) \rightarrow \alpha, \quad v^{\prime}(t) \rightarrow 0 \quad \text { as } \quad t \rightarrow-\infty
\end{array}\right.
$$

In particular, $v$ is convex and strictly increasing, so that $e^{u}-1 \geq e^{\alpha}-1>0$. Hence there is a constant $\delta>0$ such that $v^{\prime \prime} \geq \delta e^{2 t} e^{v}$ or with $w=2 t+v$,

$$
w^{\prime \prime} \geq \delta e^{w} \quad \text { on }(-\infty, T(\alpha))
$$

the maximal interval of existence for $v$. We now show $T(\alpha)<\infty$. For convenience, we assume $T(\alpha)>0$.

Multiplying (4.6) by $w^{\prime}>0$ and integrating over $(-\infty, t)(t<T(\alpha))$ lead to the inequality $\left(w^{\prime}(t)\right)^{2}>2 \delta e^{w(t)}+4$ or

$$
w^{\prime}(t)>\sqrt{2 \delta}\left(1+\frac{1}{8}[w(t)]^{2}\right) \text { for } t>0,
$$

because $w(t)>0$ when $t>0$. It is then standard from (4.7) that $w(t)$ blows up in finite time and $T(\alpha)$ is necessarily finite.

Returning to the $r$ variable, we see that $v(r)$ exists on $[0, R(\alpha))$ where $R(\alpha)=e^{T(\alpha)}$ and that $v(r) \rightarrow \infty$ as $r \rightarrow R=R(\alpha)$.

To prove the lemma, it suffices to show that $u \leq 0$ since then $u<0$ follows from the strong maximum principle.

Suppose otherwise $u>0$ at some point in $\mathbb{R}^{2}$. Without loss of generality, we assume $u(0) \equiv 2 \alpha>0$. Let $v=v(r)$ be the solution of (4.4) on $B_{R}$ (the ball in $\mathbb{R}^{2}$ with radius $R$ and centered at the origin). Since $u-v \rightarrow-\infty$ on $\partial B_{R}$ and $(u-v)(0)=\alpha>0, u-v$ has a positive interior maximum at $\tilde{x} \in B_{R}$. Therefore, with $f(\xi)=e^{\xi}\left(e^{\xi}-1\right), \xi>0, f^{\prime}(\xi) \geq 1$, we have

$$
\begin{aligned}
0 & \geq(\Delta[u-v])(\tilde{x})=\frac{4}{\kappa^{2}}[f(u(\tilde{x}))-f(v(\tilde{x}))] \\
& \geq \frac{4}{\kappa^{2}}[(u-v)(\tilde{x})] \geq \frac{4}{\kappa^{2}} \alpha>0,
\end{aligned}
$$

a contradiction.

We next determine the asymptotic behavior of $u$ by a standard potential theory argument. Note that if $u$ is already known to be radially symmetric, then the result follows as a special case of Lemma 3.6.

Lemma 4.2. Let $u \not \equiv 0$ be a $C^{2}$ solution of (4.1) satisfying (4.3). Then

$$
\frac{u(x)}{\ln |x|} \rightarrow \frac{2}{\kappa^{2} \pi} \int_{\mathbb{R}^{2}} e^{u}\left(e^{u}-1\right) d x \equiv-\beta, \quad r u_{r} \equiv x_{j} \partial_{j} u \rightarrow-\beta
$$

uniformly as $r=|x| \rightarrow \infty$, where $\beta>0$ is a constant. 
Proof. Set $f(x)=-\left(4 / \kappa^{2}\right) e^{u(x)}\left(e^{u(x)}-1\right)$. Then $f \in L\left(\mathbb{R}^{2}\right)$ and $0<f<4 / \kappa^{2}$ by virtue of Lemma 4.1.

Define

$$
v(x)=\frac{1}{2 \pi} \int_{\mathbb{R}^{2}}(\ln |x-y|-\ln |y|) f(y) d y .
$$

Then by standard arguments, $\Delta v=f$ and

$$
\frac{v(x)}{\ln |x|} \rightarrow \beta, \quad r v_{r} \rightarrow \beta \quad \text { uniformly as }|x| \rightarrow \infty .
$$

Put $h=u+v$. Then $h \leq v \leq C(\ln |x|+1)$ and $\Delta h=0$. Hence $h=$ constant and the lemma follows.

The following statement gives an estimate for the value of $\beta$ in Lemma 4.2. See also Lemma 3.6.

Corollary 4.3. There holds the inequality

$$
\pi \beta^{2} \geq 2 \pi \beta+\frac{4}{\kappa^{2}} \int_{\mathbb{R}^{2}} e^{u} d x>4 \pi \beta .
$$

Thus in particular $\beta>4$.

Proof. By the well-known Pohazaev identity, we can write for any $R>0$,

$$
\begin{aligned}
\int_{\partial B_{R}} r \frac{u_{r}^{2}}{2} d S & =\int_{\partial B_{R}} \frac{1}{r} \frac{u_{\theta}^{2}}{2} d S+\frac{4}{\kappa^{2}} \int_{B_{R}} e^{u}\left(2-e^{u}\right) d x \\
& \geq \frac{4}{\kappa^{2}} \int_{B_{R}} e^{u}\left(1-e^{u}\right) d x+\frac{4}{\kappa^{2}} \int_{B_{R}} e^{u} d x
\end{aligned}
$$

Letting $R \rightarrow \infty$ and using Lemma 4.2, we find the desired comparison (4.8).

Using the above results and the method in Chen and $\mathrm{Li}$ [7], we can now prove the main result of this section.

Theorem 4.4. Let $u$ be a solution of (4.1) and (4.3). Then $u$ is radially symmetric about some point $\tilde{x} \in \mathbb{R}^{2}$ and attains its global maximum at $x=\tilde{x}$. Moreover, $u$ is a strictly decreasing function of $r=|x-\tilde{x}|$.

Proof. It suffices to show for any direction, say the $x_{1}$-direction, that $u$ is symmetric in the direction (with respect to some choice of origin), and that $\partial_{1} u<0$ for $x_{1}>0$.

Initially we choose the coordinate system so that $\partial_{1} u(-3,0)<0$. This is possible by Lemma 4.2 and will ensure that the eventual line of symmetry $x_{1}=\lambda_{0}$ satisfies $\lambda_{0}<-3$. For $\lambda \in \mathbb{R}$, we use the notation $x=\left(x_{1}, x_{2}\right), x^{\lambda}=\left(2 \lambda-x_{1}, x_{2}\right)$, and

$$
\Sigma_{\lambda}=\left\{x \mid x_{1}<\lambda\right\}, \quad \Gamma_{\lambda}=\partial \Sigma_{\lambda}=\left\{x \mid x_{1}=\lambda\right\} .
$$

Set $w_{\lambda}(x)=u\left(x^{\lambda}\right)-u(x)$ and $\tilde{w}_{\lambda}(x)=w_{\lambda}(x) / g(x)$ with $g(x)=\ln (|x|-1)$. Both $w_{\lambda}$ and $\tilde{w}_{\lambda}$ are well-defined $(g>0)$ for $x \in \Sigma_{\lambda}$ with $\lambda<-2$.

With $f(\xi)=\left(4 / \kappa^{2}\right) e^{\xi}\left(1-e^{\xi}\right)$, a simple calculation gives

$$
\Delta w_{\lambda}+f^{\prime}(\xi(x)) w_{\lambda}=0,
$$


and so

$$
\Delta \tilde{w}_{\lambda}+\frac{2}{g} \nabla g \cdot \nabla \tilde{w}_{\lambda}+\left(f^{\prime}(\xi(x))+\frac{\Delta g}{g}\right) \tilde{w}_{\lambda}=0
$$

where $\xi(x)$ is some real number between $u(x)$ and $u\left(x^{\lambda}\right)$.

Step 1. There exists $R_{0}>0$ such that if $\tilde{x}$ is a point where $\tilde{w}_{\lambda}$ has a negative minimum, then $|\tilde{x}|<R_{0}$.

For $\xi(\tilde{x}) \leq \max \left\{u(\tilde{x}), u\left(\tilde{x}^{\lambda}\right)\right\}=u(\tilde{x})$ and

$$
\frac{\Delta g}{g}(x)=\frac{1}{|x|(|x|-1)^{2} \ln (|x|-1)}
$$

so that

$$
f^{\prime}(\xi(\tilde{x}))+\frac{\Delta g}{g}(x)<0 \quad \text { if } \quad|\tilde{x}|>R_{0}
$$

for $R_{0}$ large enough by Lemma 4.2 and Corollary $4.3(\beta>4)$. Hence $(4.10)$ and the maximum principle imply the claim.

Step 2. With $R_{0}$ as in Step $1, \tilde{w}_{\lambda}(x) \geq 0$ for $x \in \Sigma_{\lambda}, \lambda<-R_{0}$. For by Lemma 4.2, $\tilde{w}_{\lambda}(x) \rightarrow 0$ as $|x| \rightarrow \infty\left(x \in \Sigma_{\lambda}\right)$ and trivially $\tilde{w}_{\lambda}=0$ on $\Gamma_{\lambda}$. Hence if $\tilde{w}_{\lambda}<0$ at any point, $\tilde{w}_{\lambda}$ has a negative minimum at some $\tilde{x}$ with $|\tilde{x}|>R_{0}$, contradicting Step 1 .

Step 3. Define $\lambda_{0}$ to be the largest $\lambda$ such that $\tilde{w}_{\lambda}(x) \geq 0$ for $x \in \Sigma_{\lambda}$ and $\lambda \leq \lambda_{0}$. Then

a) $w_{\lambda}(x)>0$ for $x \in \Sigma_{\lambda}, \lambda<\lambda_{0}$ and $\partial_{1} u(x)>0$ for $x \in \Sigma_{\lambda_{0}}$.

b) $w_{\lambda_{0}} \equiv 0$ for $x \in \Sigma_{\lambda_{0}}$.

To prove a), observe that $w_{\lambda} \geq 0$ implies $\partial_{1} u \geq 0$ for $x_{1} \leq \lambda_{0}$ and $w_{\lambda}(x) \equiv 0$ if and only if $\partial_{1} u=0$ somewhere on $\Gamma_{\lambda}$ by the maximum principle and the Hopf Lemma.

Now suppose there is $\delta>0$ such that $w_{\lambda_{0}-\delta}(x) \equiv 0$. Then $\partial_{1} u\left(x_{1}, x_{2}\right)=0$ for $\lambda_{0}-2 \delta \leq x_{1} \leq \lambda_{0}$ and so $\partial_{1} u=0$ on $\Gamma_{\lambda_{0}-2 \delta}$ and thus $w_{\lambda_{0}-2 \delta} \equiv 0$. Repeating this argument we would have $\partial_{1} u \equiv 0$ for $x_{1}<\lambda_{0}$, contradicting Lemma 4.2. Thus $w_{\lambda}(x)>0$ for $x \in \Sigma_{\lambda}, \lambda<\lambda_{0}$. Then by the Hopf Lemma, $\partial_{1} w_{\lambda}=-2 \partial_{1} u>0$ on $\Gamma_{\lambda}$ for $\lambda<\lambda_{0}$. Thus Claim a) is proved.

To prove Claim b) we note that since $\partial_{1} u>0$ for $x_{1}<\lambda_{0}$, we must have $\lambda_{0}<-3$ by our choice of the original coordinates. Hence for $\lambda<\lambda_{0}+1$ and $x \in \Sigma_{\lambda}$, $g(x)>0$ and $\tilde{w}_{\lambda}$ is well-defined. Now suppose $w_{\lambda_{0}} \not \equiv 0$. Then by (4.9), the maximum principle, and the Hopf Lemma, we must have $w_{\lambda_{0}}>0$ in $\Sigma_{\lambda}$ and $\partial_{1} w_{\lambda_{0}}<0$ on $\Gamma_{\lambda_{0}}$. On the other hand, the definition of $\lambda_{0}$ implies there exist a sequence of $\lambda_{k} \geq \lambda_{0}$ with $\tilde{w}_{\lambda_{k}}(x)<0$ for some $x \in \Sigma_{\lambda_{k}}$. Let $x_{k}$ be points where $\tilde{w}_{\lambda_{k}}$ has a negative minimum in $\Sigma_{\lambda_{k}}$. By Step 1, $\left|x_{k}\right|<R_{0}$ and a subsequence $x_{\lambda_{k_{j}}} \rightarrow \tilde{x} \in \mathbb{R}^{2}$. Clearly, $\tilde{x} \in \Sigma_{\lambda_{0}} \cup \Gamma_{\lambda_{0}}, \tilde{w}_{\lambda_{0}}(\tilde{x}) \leq 0$, and $\nabla \tilde{w}_{\lambda_{0}}(\tilde{x})=0$. This is a contradiction.

Applying Theorem 4.4 to the Bogomol'nyi system (2.5) via (4.1), we see that finite energy non-topological solutions are all radially symmetric about some points in $\mathbb{R}^{2}$. Moreover, if $(\phi, A)$ is such a solution, then $|\phi(x)|^{2}$ attains its global maximum at its point of symmetry, say $\tilde{x},|\phi|^{2}$ is strictly decreasing with respect to $r=|x-\tilde{x}|$, and $|\phi(\tilde{x})|^{2}<1$. Therefore the first part of Theorem 2.2 is proved. 


\section{Existence of Bare Solutions}

In this section we complete the proof of Theorem 2.2 by showing that, for any $\tilde{x} \in \mathbb{R}^{2}$, (2.5) has a continuous family of bare solutions which are radially symmetric about $\tilde{x}$ and satisfy (2.9)-(2.10). Since (2.5) is invariant under space translations, we may assume $\tilde{x}=0$ and consider the corresponding radially symmetric solutions of (4.1). In this situation, we need to make a solution $u$ achieve its global maximum at $x=0$. Thus $\left(\partial_{j} u\right)(0)=0$. This motivates the initial condition for the radial version of Eq. (4.1):

$$
\left\{\begin{aligned}
u_{r r}+\frac{1}{r} u_{r} & =\frac{4}{\kappa^{2}} e^{u}\left(e^{u}-1\right), \quad r>0, \\
u(0) & =-\alpha, \\
u_{r}(0) & =0 .
\end{aligned}\right.
$$

In the proof of Lemma 4.1, we have seen that (5.1) has no global solution if $\alpha<0$. If $\alpha=0$, the only solution is the trivial one $u \equiv 0$. However, if $\alpha>0$ we have

Lemma 5.1. For any $\alpha>0$, the problem (5.1) has a unique global solution $u(r)$. This solution satisfies $u(r)<0, u_{r}(r)<0$, and is strictly concave when $r>0$. Moreover, $u(r)$ also fulfills all the properties stated in Lemma 4.2.

Proof. Let $u(r)$ be the unique local solution of (5.1). Then in the trivial of existence, there holds

$$
r u_{r}(r)=\int_{0}^{r} \varrho e^{u(\varrho)}\left(e^{u(\varrho)}-1\right) d \varrho .
$$

Using the proof of Lemma 3.4, we see that for all $r>0$ for which $u(r)$ exists, we have $u(r)<0$. Therefore $u_{r}(r)<0$ also holds. Hence $u(r)$ and $u_{r}(r)$ remain finite for all $r>0$ and the global existence follows in particular.

It is again convenient to use the substitution (3.11). Now the equation becomes (3.12) with $t_{0}=-\infty$. Thus the concavity of $u$ is clearly seen from the property $u<0$. Using the proof of Lemmas 3.4 and 3.6, we easily find

$$
\lim _{t \rightarrow \infty} u(t)=-\infty, \quad \lim _{t \rightarrow \infty} u^{\prime}(t)=-\beta \quad \text { for some } \beta>4 .
$$

Returning to the variable $r=e^{t}$, we see that our solution $u$ verifies all the statements in Lemma 4.2.

From each solution $u$ produced in Lemma 5.1 we can construct a solution pair $(\phi, A)$ of $(2.5)$ as in Sect. 3. As a consequence, $|\phi|^{2}=e^{u}$ is a decreasing function of $r=|x|$. In particular (2.9) follows (with $\tilde{x}=0$ ). Finally, by virtue of the discussion in Sect. 3 and $\beta>4$ in (5.2), we see that (2.10) can also be fulfilled with some suitable $\sigma>4$. Such a solution is of course of finite energy. This concludes the proof of Theorem 2.2.

\section{Further Remarks}

In this paper we have proved the existence of radially symmetric non-topological $N$-vortex solutions in the self-dual Chern-Simons model: The vortices are all superimposed at one point. The existence of non-topological solutions with an arbitrary 
vortex-line distribution is still an open question. We would also like to call attention to the following problems.

i) We were unable to decide the exact value of the decay exponent $\sigma=\sigma(\alpha)$ in (2.8) or (2.10). In fact we could not decide the value of the $\beta>2 N+4$ in (3.24) or $\beta>4$ in (5.2) which is the sharp upper bound for $\sigma$. At this moment, it seems impossible for example to find the true value of $\beta$ from (3.26) because the right-hand side of the equation is in general unknown. However, $\beta$ is an important physical quantity which yields the values of charge, energy, and magnetic flux of the solutions. For instance, using (2.5), (3.14), (3.17), (3.24) [or (5.2)], we see that the flux $\Phi$ takes the form

$$
\begin{aligned}
\Phi & =\int_{\mathbb{R}^{2}} F_{12} d x=-\frac{2}{\kappa^{2}} \int_{\mathbb{R}^{2}}|\phi|^{2}\left(|\phi|^{2}-1\right) d x \\
& =-\frac{4 \pi}{\kappa^{2}} \int_{0}^{\infty} r e^{u}\left(e^{u}-1\right) d r=-\frac{4 \pi}{\kappa^{2}} \int_{-\infty}^{\infty} e^{2 t} e^{u}\left(e^{u}-1\right) d t \\
& =-\pi\left[\lim _{t \rightarrow \infty} u^{\prime}(t)\right]+\pi\left[\lim _{t \rightarrow-\infty} u^{\prime}(t)\right]=(2 N+\beta) \pi,
\end{aligned}
$$

for $N=0,1,2, \ldots$ It is interesting to compare this result with the flux of a topological $N$-vortex solution for which $\Phi=2 \pi N$. Since $\beta>2 N+4$, therefore a non-topological $N$-vortex solution carries more than two times magnetic flux as that for a topological solution. Our estimate $\Phi>4(N+1) \pi$ also slightly improves the earlier conclusion $\Phi \geq 4(N+1) \pi$ reached in [12-14].

ii) We only proved that any solution of (4.1) under the condition (4.3) must be radially symmetric. An interesting open question is: Should a solution $v$ of (3.3) be radially symmetric about the origin if $\int_{\mathbb{R}^{2}}|x|^{2 N} e^{v}\left(1-|x|^{2 N} e^{v}\right) d x<\infty$ ?

iii) It is not known whether the Chern-Simons model (2.1) allows vortex condensates. (In the Yang-Mills systems, such solutions do occur. For example, in the classical electroweak theory $[1,2,23]$.) This question is equivalent to the solvability of the equation

$$
\Delta u=\frac{4}{\kappa^{2}} e^{u}\left(e^{u}-1\right)+4 \pi \sum_{j=1}^{N} \delta_{p_{j}},
$$

over a two-torus $M$ (see $[20,26])$. It is easily seen that a necessary condition for the existence of a solution to (6.1) is [26]

$$
N<\frac{|M|}{4 \pi \kappa^{2}}
$$

However, it is not clear whether (6.2) is also sufficient. Actually no sufficient condition for the solvability of (6.1) has been found yet.

\section{References}

1. Ambjorn, J., Olesen, P.: A magnetic condensate solution of the classical electroweak theory. Phys. Lett. B 218, 67-71 (1989)

2. Ambjorn, J., Olesen, P.: On electroweak magnetism. Nucl. Phys. B 315, 606-614 (1989)

3. Berestycki, H., Lions, P.L., Peletier, L.A.: An ODE approach to the existence of positive solutions for semilinear problems in $\mathbb{R}^{N}$. Indiana Univ. Math. J. 30, 141-157 (1981) 
4. Berestycki, H., Nirenberg, L.: On the method of moving planes and the sliding method. Preprint

5. Bogomol'nyi, E.B.: The stability of classical solutions. Sov. J. Nucl. Phys. 24, 449-454 (1976)

6. Caffarelli, L., Gidas, B., Spruck, J.: Asymptotic symmetry and local behavior of semilinear elliptic equations with critical Sobolev growth. Preprint

7. Chen, W., Li, C.: Classification of solutions of some nonlinear elliptic equations. Preprint

8. Gidas, B., Ni, W.-M., Nirenberg, L.: Symmetry of positive solutions of nonlinear elliptic equations in $\mathbb{R}^{n}$. Mathematical Analysis and Applications, Part A. Adv. Math. Suppl. Studies 7A, pp. 369-402. L. Nachbin (ed.), New York: Academic Press 1981

9. Gidas, B., Spruck, J.: Global and local behavior of positive solutions of nonlinear elliptic equations. Commun. Pure Appl. Math. 34, 528-598 (1981)

10. Hong, J., Kim, Y., Pac, P.Y.: Multivortex solutions of the abelian Chern-Simons theory. Phys. Rev. Lett. 64, 2230-2233 (1990)

11. Jackiw, R.: Solitons in Chern-Simons/anyon systems. Preprint (Massachusetts Institute of Technology, 1991)

12. Jackiw, R., Lee, K., Weinberg, E.J.: Self-dual Chern-Simons solitons. Phys. Rev. D 42, 34883499 (1990)

13. Jackiw, R., Pi, S.-Y.: Self-dual Chern-Simons solitons. Preprint (Massachusetts Instuitute of Technology, 1991)

14. Jackiw, R., Pi, S.-Y., Weinberg, E.J.: Topological and non-topological solitons in relativistic and non-relativistic Chern-Simons theory. Preprint (Columbia University, 1990)

15. Jackiw, R., Weinberg, E.J.: Self-dual Chern-Simons vortices. Phys. Rev. Lett. 64, 2234-2237 (1990)

16. Jaffe, A., Taubes, C.H.: Vortices and Monopoles. Boston: Birkhäuser 1980

17. Lee, K.: Self-dual nonabelian Chern-Simons solitons. Phys. Rev. Lett. 66, 553-555 (1991)

18. Li, C.: Monotonicity and symmetry of solutions of fully nonlinear equations I, II.: bounded domains, unbounded domains. Commun. P.D.E. (to appear) 19. Liouville, J.: Sur l'équation aux différences partielles $\frac{d^{2} \log \lambda}{d u d v} \pm \frac{\lambda}{2 a^{2}}=0$. J. Math. Pures Appl.
18, 71-72 (1853)

20. Olesen, P.: Soliton condensate in some self-dual Chern-Simons theories. Preprint (The Niels Bohr Institute, 1991)

21. Protter, M.H., Weinberger, H.F.: Maximum Principles in Differential Equations. New Jersey: Prentice-Hall 1967

22. Spruck, J., Yang, Y.: Topological solutions in the self-dual Chern-Simons theory. Preprint

23. Spruck, J., Yang, Y.: On multivortices in the electroweak theory. I: Existence of periodic solutions. Commun. Math. Phys. 144, 1-16 (1992)

24. Tyupkin, Yu., Fateev, V.A., Shvarts, A.S.: Particle-like solutions of the equations of gauge theories. Theor. Math. Phys. 26, 270-273 (1976)

25. Wang, R.: The existence of Chern-Simons vortices. Commun. Math. Phys. 137, 587-597 (1991)

26. Yang, Y.: A generalized self-dual Chern-Simons Higgs theory. Lett. Math. Phys. 23, 179-191 (1991)

Communicated by A. Jaffe 